\title{
A MATHEMATICAL INVESTIGATION OF DIFFERENT GROWTH RATES IN SIMULTANEOUS TUMORS AT TWO DISTANT SITES
}

\author{
by \\ Farjana Aktar \\ Bachelor of Science in Statistics, Dhaka University, 2004 \\ Masters of Science in Statistics, Dhaka University, 2005
}

\author{
A thesis \\ presented to Ryerson University \\ in partial fulfillment of the \\ requirements for the degree of \\ Master of Science \\ in the program of \\ Applied Mathematics \\ Toronto, Ontario, Canada, 2019 \\ (C)Farjana Aktar, 2019
}




\section{Author's Declaration}

I hereby declare that I am the sole author of this thesis. This is a true copy of the thesis, including any required final revisions, as accepted by my examiners.

I authorize Ryerson University to lend this thesis to other institutions or individuals for the purpose of scholarly research.

I further authorize Ryerson University to reproduce this thesis by photocopying or by other means, in total or in part, at the request of other institutions or individuals for the purpose of scholarly research.

I understand that my thesis may be made electronically available to the public. 


\title{
A MATHEMATICAL INVESTIGATION OF DIFFERENT GROWTH RATES IN SIMULTANEOUS TUMORS AT TWO DISTANT SITES
}

\author{
Master of Science, 2019 \\ Farjana Aktar \\ Applied Mathematics, Ryerson University
}

\begin{abstract}
Experimental data demonstrates that simultaneous injection of cancer cells at two distinct sites often results in one large and one small tumour. Unbalanced tumour-stimulating inflammation is hypothesized to be the cause of this growth rate separation, causing one tumour to grow faster than the other. Here, a mathematical model for immune recruitment and competition between two cancer sites is developed to explore the role of tumour-promoting inflammation in the observed growth rate separation. Due to the experimental set-up, immune predation may be neglected, focusing the model on tumour-promoting immune actions. A new mathematical model with localized immune recruitment and competition between the two cancer sites is developed using a multi-compartment ODE system. A simulated annealing algorithm is used to fit the model to control data (one tumour burden). Stability and parameter sensitivity analyses are used to explore the mathematical model and parameter space. Next, the twotumour scenario is predicted by testing parameter values tied to possible biological mechanisms of action. The model predicts that indeed inflammation may be a contributor to growth rate separation observed in simultaneous tumour growth, if one site is pre-inflamed compared to the other.
\end{abstract}




\section{Acknowledgements}

First and foremost, I offer my humble thanks to Allah for giving me the opportunity, strength, and guidance to continue and have finished my master's degree.

Secondly, I would like to sincerely express deep gratitude to my supervisor, Dr. Kathleen Wilkie, for her valuable assistance, counseling, support, patience, and kindness. Without her help, I would not have been able to finish my thesis. Words are not enough to express how grateful I am. I owe her much.

I would also like to give special thanks to my other honorable teachers, who are the readers and examiners, Dr. Katrin Rohlf, Dr. JP Pascal, and Dr. Konstantanos Georgiou who gave their valuable time to finish my achievement successfully.

Most importantly, I would like to give my special thanks to my husband, Enayet Hossain, for his support and patience, and also my two lovely daughters whose love and sacrifice have sustained me during my study.

I also would like to give humble thanks to my parents, and my all sisters who have given me the mental strength, guidance, and encouragement to continue my studies.

Finally, I would like to thank my friend, Meghna, and elder sister, Shyla Ahmed, who are my inspirational ladies who always gives me support at all times. 


\section{Dedication}

I would like to dedicate this thesis to my husband, Enayet Hossain, who leads me through the valley of darkness with light of hope and support. 


\section{Table of Contents}

Author's Declaration $\quad$ ii

$\begin{array}{ll}\text { Abstract } & \text { iii }\end{array}$

Acknowledgements $\quad$ iv

$\begin{array}{lll}\text { Dedication } & \text { v }\end{array}$

List of Tables $\quad$ viii

$\begin{array}{ll}\text { List of Figures } & \text { ix }\end{array}$

1 Introduction $\quad 1$

1.1 Cancer and Inflammation . . . . . . . . . . . . . . . . . 2

1.2 Mathematical Models . . . . . . . . . . . . . . . . . . . . . 2

1.3 Concomitant Resistance . . . . . . . . . . . . . . . . . . . . . 3

2 Experimental Data and Data Analysis $\quad 6$

3 Model Equations $\quad 12$

3.1 Hypothesis . . . . . . . . . . . . . . . . . . . . . . . 12

3.2 Control Tumour Model . . . . . . . . . . . . . . . . . . . . . 12

3.3 Two-Tumour Model . . . . . . . . . . . . . . . . . . . . . 13

4 Model Parameterization $\quad 15$

5 Model Analysis $\quad 19$

5.1 Model Steady State Analyses . . . . . . . . . . . . . . . . . . . . 19

5.2 Parameter Sensitivity . . . . . . . . . . . . . . . . . 21 
6 Results $\quad \mathbf{2 2}$

6.1 Single Variable Mechanisms . . . . . . . . . . . . . . . . . . 22

6.2 Combination Mechanisms . . . . . . . . . . . . . . . 25

7 Discussion and Conclusions $\quad 28$

7.1 Future Work . . . . . . . . . . . . . . . . . . 30

$\begin{array}{ll}\text { References } & 33\end{array}$ 


\section{List of Tables}

2.1 Exponential and logistic growth models to be fit to the experimental data. . . . 7

2.2 Summary table of the estimated parameter values for the growth rate analysis. . 11

4.1 Simulated annealing results from ten runs of the algorithm to fit the one-tumour model parameters $\alpha, K_{0}, p, q, \beta$, and $\rho$ to control experimental data. The minimum RMSE parameter set (run 4) is highlighted in red. Run 4 parameter values were rounded to three significant digits for use in all numerical simulations (Final Set). . . . . . . . . . . . . . . . . . 17

4.2 Baseline fitted and fixed parameter values used in all numerical simulations unless otherwise stated. . . . . . . . . . . . . . . . . . 18 


\section{List of Figures}

2.1 Experimental data for the control group and experimental group divided into big and small tumours. . . . . . . . . . . . . . . . . . . . .

2.2 Control tumour data fit with the exponential model assuming either a fixed or fitted initial condition. . . . . . . . . . . . . . . . . . . . . 8

2.3 Control tumour data fit with the logistic model assuming either a fixed or fitted initial condition. . . . . . . . . . . . . . . . . 8

2.4 Small tumour data fit with the exponential model assuming either a fixed or fitted initial condition. . . . . . . . . . . . . . . . . . . . 9

2.5 Small tumour data fit with the logistic model assuming either a fixed or fitted initial condition. . . . . . . . . . . . . . . . . . . . . 9

2.6 Big tumour data fit with the exponential model assuming either a fixed or fitted initial condition. . . . . . . . . . . . . . . . . . . . 10

2.7 Big tumour data fit with the logistic model assuming either a fixed or fitted initial condition. . . . . . . . . . . . . . . . . . . . 10

4.1 The best fit from the simulated annealing algorithm (run 4) numerically plotted against the control experimental data. . . . . . . . . . . . . . . 17

4.2 Ten runs of the simulated annealing algorithm produced ten parameter sets that all fit the control data equally well (see Table 4.1). The set with the smallest RMSE, rounded to three significant digits, was chosen to work with in all future simulations (Final Set in Table 4.1).

5.1 The dependence of equilibrium tumour volume, $K_{C}^{e q}$, in the control model, on parameters $a$ and $b$, shown in (a); and the dependence of equilibrium tumour volumes $K_{C 1}^{e q}$, and $K_{C 2}^{e q}$, of the two-tumour model, on immune equilibrium size $I_{1}^{e q}$, shown in (b) . . . . . . . . . . . . . . . 20

5.2 Each parameter value is varied by $20 \%$ up and down and the relative change in tumour volume at day 15 is reported. 
6.1 Two-tumour simulation testing differences in initial tumour size (a) and intrinsic immune growth rate $(\mathrm{b}) \ldots \ldots \ldots \ldots \ldots$

6.2 Two-tumour simulation testing differences in initial immune size (a) and immune recruitment (b) . . . . . . . . . . . . . . . . . . . . . . . . 24

6.3 Two-tumour simulation varying either the four parameters $C_{20}, I_{10}, \beta_{2}$, and $\rho_{2}$ in (a), or varying the initial carrying capacities $K_{10}$ and $K_{20}$ in (b). . . . . . . 25

6.4 Two-tumour simulation varying either the six parameters $C_{20}, I_{10}, \beta_{2}, \rho_{2}, K_{10}$, and $K_{20}$ in (a), or varying the pre-inflamed parameters $I_{10}, I_{20}, K_{10}$, and $K_{20}$ in

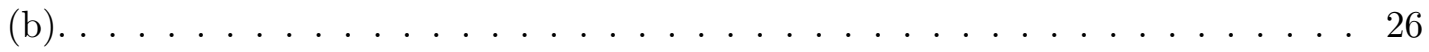




\section{Chapter 1}

\section{Introduction}

Inflammation is a powerful promoter of tumour growth that can cause unexpected tumour growth dynamics [23]. In this thesis, a mathematical model for inflammation-driven tumour growth is developed and analyzed, in an attempt to explain the surprising growth rate separation observed in simultaneously injected tumours in mice.

In an interesting laboratory experiment, it was found that mice bearing two tumours (from equal injections given on the same day) consistently grew one large and one small tumour [7]. In the originating article [7], the data was analyzed through simulations of several mathematical models for concomitant resistance. Their final proposed hypothesis was that both tumours produce inhibitory signals that act to suppress tumour growth systemically. Then, given an initially small difference in tumour volume, growth suppression would proportionally affect the smaller tumour over the larger one, leading to the observed growth rate differences.

Contrasting the concomitant resistance theory, this thesis proposes that tumour-promoting inflammation preferentially drawn to one site over the other may explain the different growth rates. Unbalanced immune recruitment and positive feedback loops in the signaling networks [23] may result in a larger accumulation of immune cells in one site over the other. This thesis will explore this hypothesis mathematically to test whether unbalanced pro-tumour immune actions can explain the observed growth phenomenon.

\subsection{Cancer and Inflammation}

Cancer is a disease characterized by uncontrolled cell growth that can form tumour masses in tissue. Cancer cells can move throughout the body via the blood or lymphatic systems, and they can initiate the growth of new blood vessels through angiogenesis. A highly vascularized tumour will most likely have a large blood and nutrient supply as well as inflammatory immune 
cells which mainly act to support tumour growth $[23,4,16]$.

Cancers have been called wounds that never heal. Inflammation, a major component of the wound healing process, is activated by tissue damage (such as that caused by tumour growth) [16]. The tissue damage and cell death caused in tumours triggers a chronic inflammatory environment. This chronic state promotes the growth and spread of cancer by increasing angiognesis, remodelling the surrounding tissue, bringing nutrients and naive immune cells to the tumour site, producing tumour growth factors, and generally increasing blood flow [16, 23].

Cancer cells can produce signals that reprogram surrounding tissues to perform actions that support their growth [26]. They can distort immune signals to convert any anti-tumour actions into pro-tumour actions, deactivate cytotoxic immune cells, and amplify and mimic recruitment signals $[16,23,13]$. As the immune system interacts with cancer cells, it may sculpt the cell phenotype, developing a less immunogenic variant that facilitates tumour growth and immune evasion [13].

The cytokine and chemokine expression profile of the tumour microenvironment may be more relevant than its specific immune cell content $[23,16]$. Cytokines can either promote or inhibit tumour development and progression, regardless of their source, by acting in concert to selectively polarize immune cells [21]. That is, the actions of immune cells within the tumour microenvironment are determined by the cytokine milieu present at the moment the naive cell arrives.

\subsection{Mathematical Models for Immuno-Oncology}

Despite the mathematical modeling approach used to describe cancer-immune interactions, all model simulations will invariably predict only three possible tumour outcomes: tumour escape (obtaining large mass sizes), tumour elimination (being cleared from the host), and tumour dormancy (sustaining periods of non-growth) [28].

Ordinary differential equation (ODE)-based models are an important framework used to describe cancer-immune interactions because they are relatively simple to develop, analyze, and numerically simulate compared to other mathematical techniques. In the literature, the majority of cancer-immune research uses ODE models [19, 10, 18, 9, 11, 12, 14, 27, 28, 29]. Some excellent reviews on this subject include a review of non-spatial time-varying models [14] and a review of dormant or near-dormant tumour states [27]. Other mathematical methods include stochastic differential equation-based models [20], models based on the kinetic theory for active particles $[1,3]$, spatio-temporal partial differential equation-based models $[22,24,2,6]$, and cellular automaton models $[24,15]$.

In mathematical descriptions of tumour growth, either exponential growth, Gompertzian 
growth, or logistic growth are assumed. For early time analyses, exponential growth is sufficient. However, for late-stage analyses, Gompertzian or logistic growth models are preferred because of the self-limiting property of these curves. The limiting size of the mass is termed the carrying capacity, and it is typically assumed to be constant. The capacity refers to the tumour microenvironment's ability to support the tumour mass. Biologically, it then makes sense that this capacity should increase as the tumour mass increases. Hahnfeldt et al [17] proposed a mathematical model for a dynamically growing carrying capacity, which is incorporated into the model developed in this thesis, as it is more biological.

\subsection{Concomitant Resistance}

As mentioned earlier, one possible explanation for the growth rate differences in the two-tumour bearing mice is that of concomitant resistance [7]. Concomitant resistance is the phenomenon where the presence of one tumour negatively influences the growth of another [25]. Several mechanisms have been proposed to explain the phenomenon, but no consensus has been reached [25]. Strong evidence for concomitant resistance is the noticeable growth rate increase of metastases upon resection of the primary tumour [25]. Some possible mechanisms to explain concomitant resistance include: (1) competition for nutrients between tumour sites, (2) production of antiangiogenic factors by the tumour masses, and (3) production of anti-proliferative factors by the tumour masses [7].

Another possible mechanism that can cause concomitant resistance is concomitant immunity [5]. This mechanism is generated by an anti-tumour immune response that is strong enough to suppress small masses while not significantly altering the growth of the large tumour mass. However, in the experimental data used in this thesis, the tumours are non-immunogenic, meaning they do not generate a significant anti-tumour immune response. Thus, concomitant immunity cannot be applied to explain the observed growth phenomenon.

Benzekry et al. [7] present several mathematical models to explore concomitant resistance as a potential mechanism driving the observed growth rate differences in the studied experimental data. They examine three mechanisms of action: (1) competition for nutrients, (2) angiogenesis inhibition, and (3) proliferation inhibition. Models are composed of systems of ordinary differential equations. By comparing numerical simulations to the experimental data, they conclude that the proliferation-inhibition model is the most likely mechanism to explain the growth separation observed.

The proliferation-inhibition model describes the growth dynamics of two tumours, where each tumour is divided into proliferative (dividing) and quiescent (non-dividing) compartments [7]. The proliferative cells are assumed to grow exponentially and to produce factors that act 
both locally and systemically to transition proliferative cells into quiescent cells. Quiescent cells are assumed to stay quiescent, i.e. they cannot transition back to the proliferative compartment. Their proposed model is below. Here $P_{i}$ and $Q_{i}$ are the proliferative and quiescent compartments, respectively, for each tumour $i=1,2$. The total tumour volume for each site is the sum of the proliferative and quiescent compartments, $V_{i}=P_{i}+Q_{i}$, for $i=1,2$. Below, $\alpha$ is the exponential growth rate of the proliferative compartments, $\beta$ is the rate at which proliferative cells transition to quiescent cells due to local signals, and $\gamma$ is the rate at which proliferative cells transition to quiescent cells due to systemic signals deriving from both tumour sites.

$$
\begin{aligned}
\frac{d P_{1}}{d t} & =\alpha P_{1}-\left(\beta P_{1}+\gamma\left(P_{1}+P_{2}\right)\right) 1_{p_{1}>0} & & P_{1}(0)=V_{0,1} \\
\frac{d Q_{1}}{d t} & =\left(\beta P_{1}+\gamma\left(P_{1}+P_{2}\right)\right) 1_{p_{1}>0} & & Q_{1}(0)=0 \\
\frac{d P_{2}}{d t} & =\alpha P_{2}-\left(\beta P_{2}+\gamma\left(P_{1}+P_{2}\right)\right) 1_{p_{2}>0} & & P_{2}(0)=V_{0,2} \\
\frac{d Q_{2}}{d t} & =\left(\beta P_{2}+\gamma\left(P_{1}+P_{2}\right)\right) 1_{p_{2}>0} & & Q_{2}(0)=0 \\
V_{1} & =P_{1}+Q_{1} & & \\
V_{2} & =P_{2}+Q_{2} & &
\end{aligned}
$$

Here the transition from the proliferative compartment to the quiescent compartment is controlled by the Heaviside function $1_{P_{i}>0}$, for $i=1$, 2. These functions are equal to one if $P_{i}>0$ and zero otherwise. This enforces the fact that when factors are present but no proliferative cells exist at that site, there is no transition into quiescence.

This thesis presents an alternative hypothesis to the proliferative-inhibition theory of concomitant resistance, to explain the observed growth rate differences in the experimental data. Proliferation-inhibition requires one tumour to be larger than the other, and this is modelled via different initial conditions $\left(V_{0,1}>V_{0,2}\right)$. As will be seen in this work, the developed model can predict the data with equal initial conditions, assuming only a pre-inflamed tumour microenvironment. This result better fits the experimental setup, and results in a simpler biological explanation.

The remainder of this thesis is outlined as follows. Chapter 2 presents and analyzes the experimental data. Chapter 3 presents the biological hypothesis to explain the different tumour growth rates and develops a mathematical model to test this hypothesis. Chapter 4 parameterizes the model using a simulated annealing algorithm, and Chapter 5 presents the results of model analyses. Chapter 6 presents numerical results of model simulations designed to test the biological hypothesis. And finally Chapter 7 concludes the thesis with a brief discussion and 
some potential future work. 


\section{Chapter 2}

\section{Experimental Data and Data Analysis}

The experimental setup is designed to compare the growth rates of tumours in C57BL/6 mice bearing either one or two cancer burdens. Both the control group (mice bearing one tumour) and the experimental group (mice bearing two tumours) consist of 10 mice.

On day 0 , the control group received one injection of $10^{6}$ Murine Lewis Lung Carcinoma (LLC) cells into the caudal half of the back. The experimental group received the same injection but on both sides $\left(10^{6}\right.$ LLC cells into each side). Full details of the experimental setup are described in [7].

In general, the experimental group consisted of mice where one tumour grew larger than the other. Two mice in this group, however, grew tumours of similar size. Upon sacrifice, these 2 mice were found to bear tumours that were directly connected by large blood vessels. Since these mice did not contain isolated tumours, they were excluded from the analysis.

Figure 2.1 shows the average tumour volumes with standard error (error bars) for the control group as well as the experimental group divided into large and small tumours. The experimental data suggests that in a mouse bearing two tumours, one grows at the control rate while the other is comparatively suppressed. This is demonstrated in Figure 2.1 where an asterisk is used to indicate statistically significant differences between the control group and the small tumours (p-values less than 0.05). No statistically significant difference was found between the control and large tumour groups.

Using the experimental data (average tumour size at discrete time points), cancer growth rates are estimated for the three tumour groups: Control, Big, and Small tumours. To do this, exponential and logistic growth models are fit to the data sets assuming either a fixed $\left(C(0)=10^{6} \approx 1 \mathrm{~mm}^{3}\right)$ or variable $\left(C(0)=C_{0}\right)$ initial condition. To measure the goodness-of- 


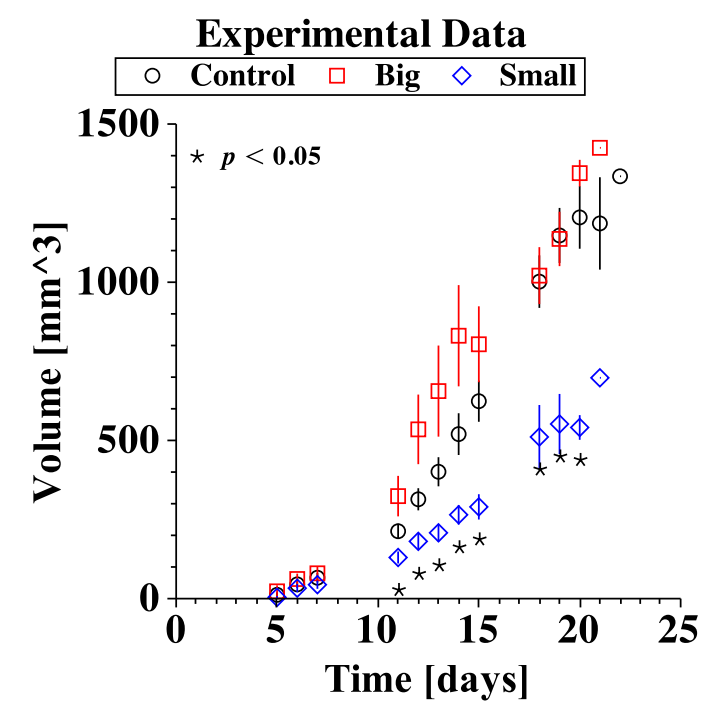

Figure 2.1: Experimental data for the control group and experimental group divided into big and small tumours.

fit, the root mean squared error is used. It is defined by RMSE $=\sqrt{\frac{1}{n} \sum_{i=1}^{n}\left(C_{i}-C\left(t_{i}\right)\right)^{2}}$ where $C_{i}$ are the tumour volume measurements on day $i$ and $C\left(t_{i}\right)$ is the model predicted tumour volume on day $t_{i}$, and $n$ is the total number of data points available.

Table 2.1: Exponential and logistic growth models to be fit to the experimental data.

\begin{tabular}{cccc}
\hline Fit 1 & Fit 2 & Fit 3 & Fit 4 \\
\hline$\frac{d C}{d t}=\alpha C$ & $\frac{d C}{d t}=\alpha C$ & $\frac{d C}{d t}=\alpha C\left(1-\frac{C}{K}\right)$ & $\frac{d C}{d t}=\alpha C\left(1-\frac{C}{K}\right)$ \\
$C_{0}=1 \mathrm{~mm}^{3}$ & $C_{0}=C 0 \mathrm{~mm}^{3}$ & $C_{0}=1 \mathrm{~mm}^{3}$ & $C_{0}=C 0 \mathrm{~mm}^{3}$ \\
\hline 1 free parameter & 2 free parameters & 2 free parameters & 3 free parameters \\
$\alpha$ & $\alpha, C_{0}$ & $\alpha, K$ & $\alpha, K, C_{0}$ \\
\hline
\end{tabular}



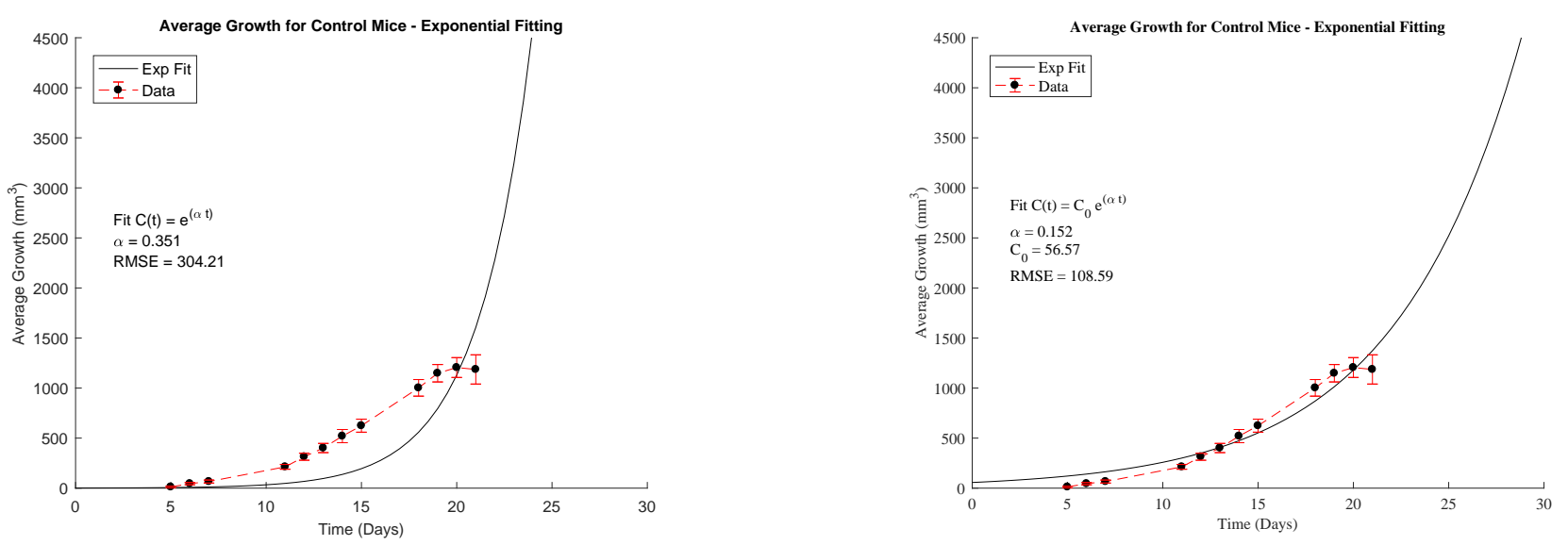

Figure 2.2: Control tumour data fit with the exponential model assuming either a fixed or fitted initial condition.
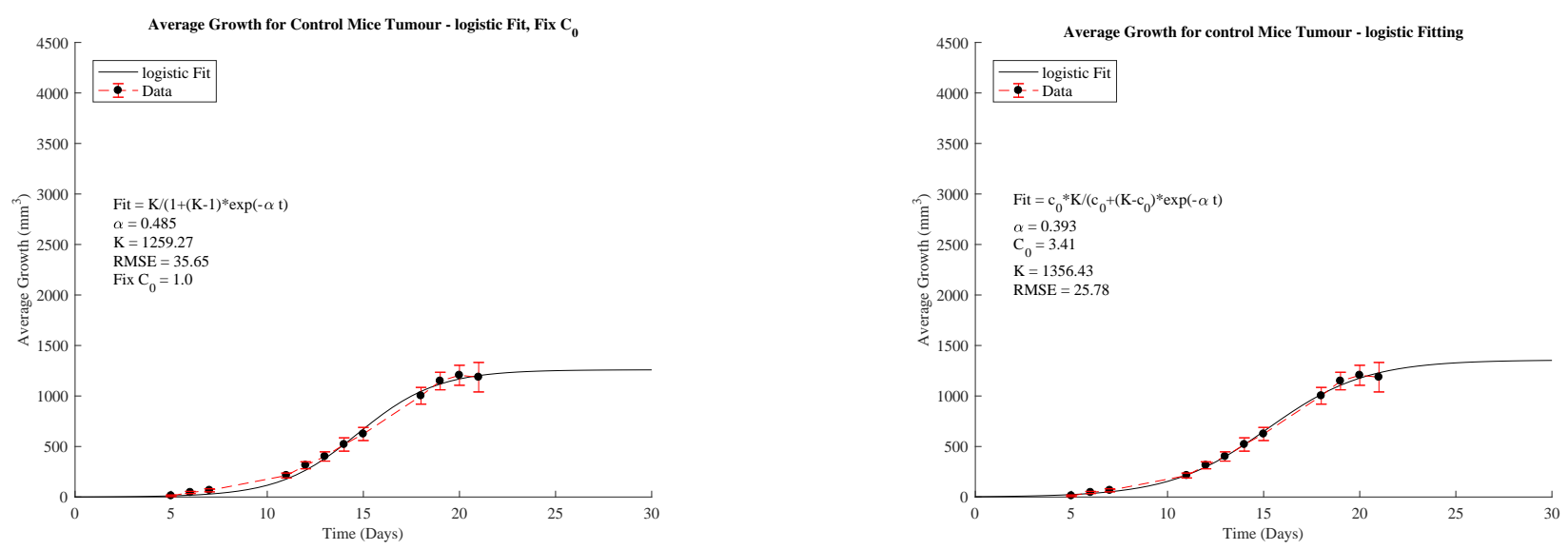

Figure 2.3: Control tumour data fit with the logistic model assuming either a fixed or fitted initial condition. 

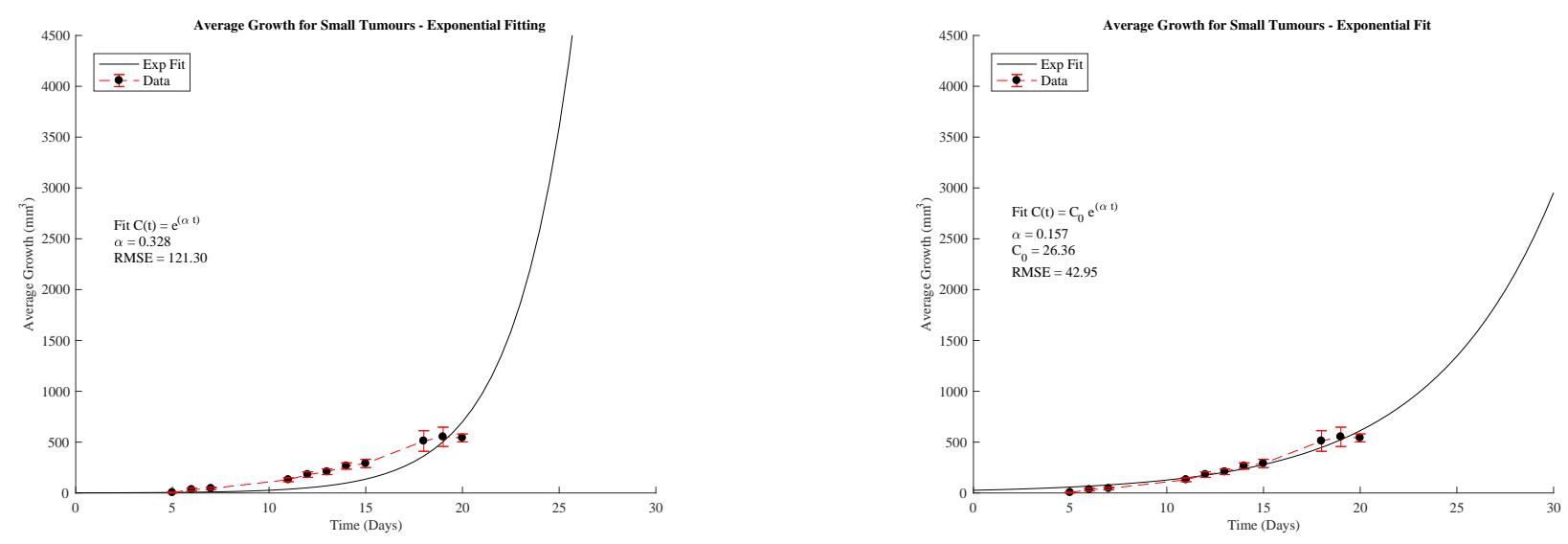

Figure 2.4: Small tumour data fit with the exponential model assuming either a fixed or fitted initial condition.
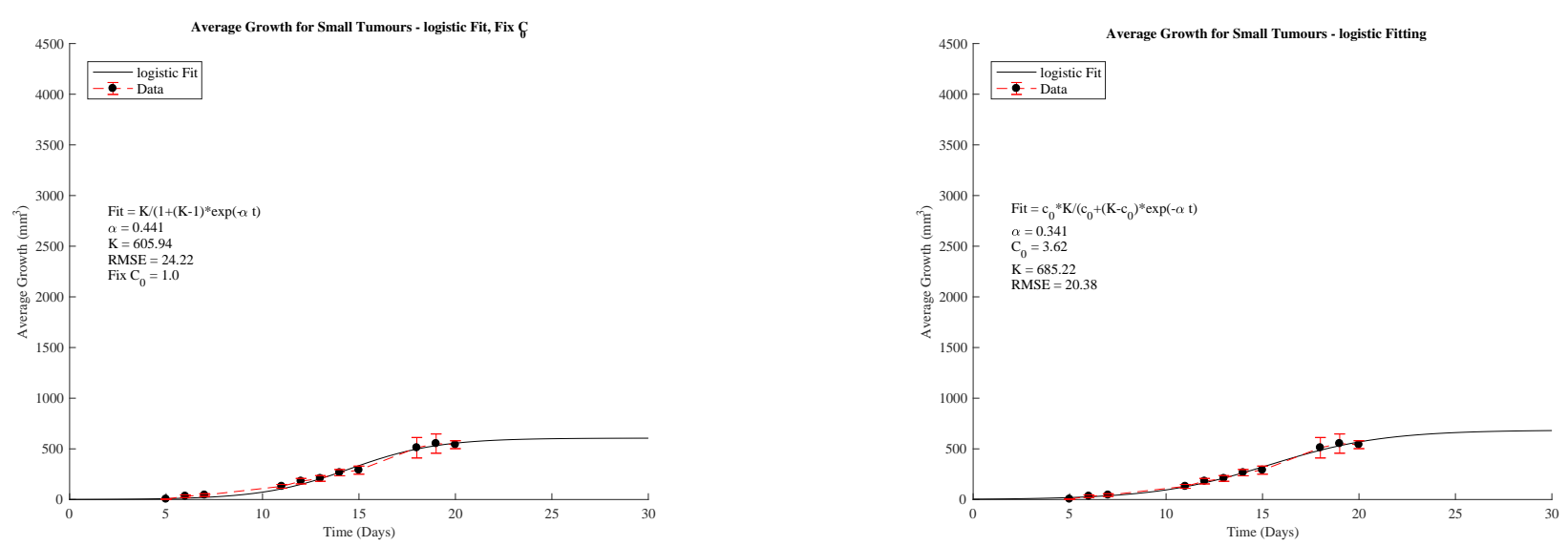

Figure 2.5: Small tumour data fit with the logistic model assuming either a fixed or fitted initial condition. 

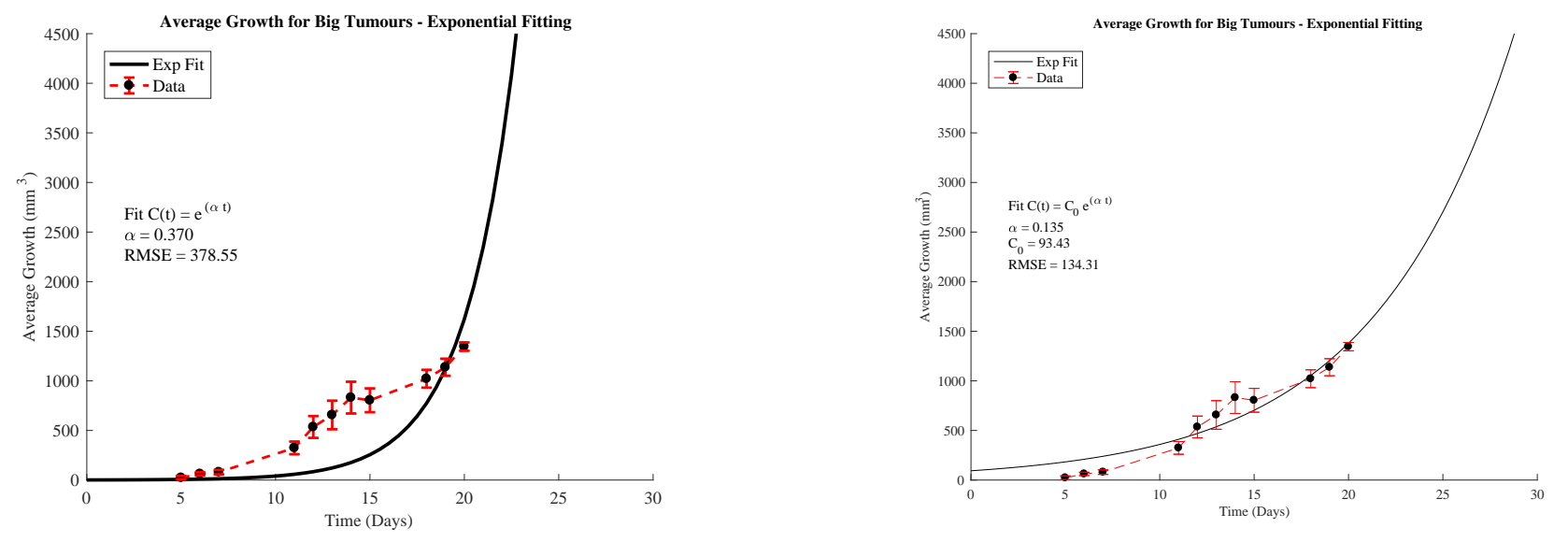

Figure 2.6: Big tumour data fit with the exponential model assuming either a fixed or fitted initial condition.
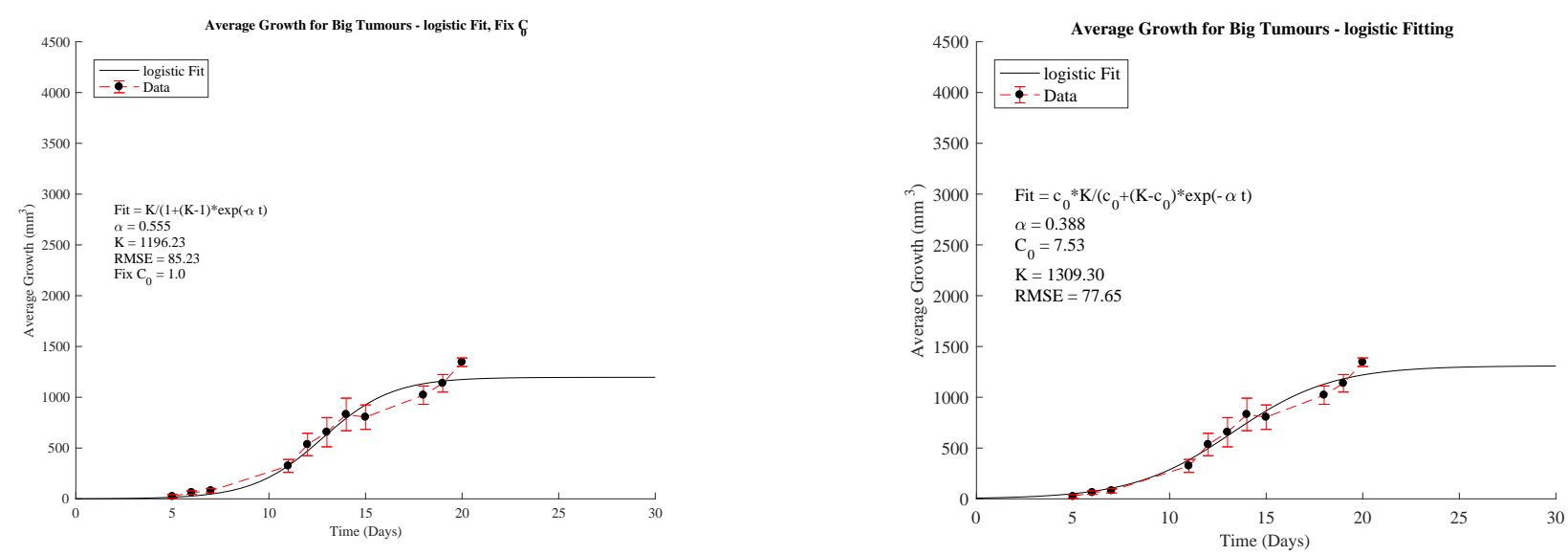

Figure 2.7: Big tumour data fit with the logistic model assuming either a fixed or fitted initial condition. 
The MATLAB fminsearch function is used to minimize the root mean squared error of the model simulations to the data points for each data group. Results of the data fitting are shown in Figures 2.2-2.7. Table 2.2 summarizes the fitted models and estimated growth rate parameters. The two parameter models (exponential with $C_{0}$ fit and logistic with $C_{0}$ fixed) both provide good fits compared to the one parameter exponential model. As expected, the overall best fit is obtained by the three parameter logistic growth model as measured by the smallest RMSE in all three tumour sizes. It is concluded that the data curves are best described by the logistic model, even when comparing the two 2-parameter models. Thus, logistic growth, rather than exponential, will be used in the model development, since it better describes the data and is a more realistic model.

Table 2.2: Summary table of the estimated parameter values for the growth rate analysis.

\begin{tabular}{l|c|c|c}
\hline Model & Small Tumour & Big Tumour & Control Tumour \\
\hline$C(t)=e^{\alpha t}, C_{0}=1$ & $\begin{array}{c}\alpha=0.328 \\
\mathrm{RMSE}=121\end{array}$ & $\begin{array}{c}\alpha=0.370 \\
\mathrm{RMSE}=379\end{array}$ & $\begin{array}{c}\alpha=0.351 \\
\mathrm{RMSE}=304\end{array}$ \\
\hline \multirow{2}{*}{$C(t)=C_{0} e^{\alpha t}$} & $\alpha=0.157$ & $\alpha=0.135$ & $\alpha=0.152$ \\
& $C_{0}=26.4$ & $C_{0}=93.4$ & $C_{0}=56.6$ \\
& $\mathrm{RMSE}=43$ & $\mathrm{RMSE}=134$ & $\mathrm{RMSE}=109$ \\
\hline \multirow{2}{*}{$C(t)=\frac{K}{(K-1) e^{-\alpha t}+1}$} & $\alpha=0.441$ & $\alpha=0.555$ & $\alpha=0.485$ \\
$C_{0}=1$ & $K=606$ & $K=1196$ & $K=1259$ \\
\hline & $\mathrm{RMSE}=24$ & $\mathrm{RMSE}=85$ & $\mathrm{RMSE}=36$ \\
\hline & $\alpha(t)=\frac{\alpha=0.341}{\left(K-C_{0}\right) e^{-\alpha t}+C_{0}}$ & $\alpha=0.388$ & $\alpha=0.393$ \\
& $K=685$ & $K=1309$ & $K=1356$ \\
& $C_{0}=3.6$ & $C_{0}=7.53$ & $C_{0}=3.41$ \\
& $\mathrm{RMSE}=20$ & $\mathrm{RMSE}=78$ & $\mathrm{RMSE}=26$ \\
\hline
\end{tabular}




\section{Chapter 3}

\section{Hypothesis and Mathematical Model}

\subsection{Hypothesis}

The hypothesis presented in this thesis proposes that inflammation may be preferentially accumulating in one site over another causing the different tumour growth rates. The positive feedback loops in pro-inflammatory signaling can cause a growth rate discrepancy between two cancer sites by preferentially accumulating at one site over the other, amplifying initially small deviations into significantly different tumour sizes. To test this hypothesis, mathematical modeling is used to determine if unbalanced pro-tumour immune actions can explain the observed growth phenomenon.

\subsection{Control Tumour Model}

In this work, cancer and immune cell populations are assumed to grow according to a logistic growth law that is mechanistically modified by their interactions. Further, the cancer population has a dynamically growing carrying capacity. A generalization of this model can be found in $[29]$.

The control one-tumour model, equations (3.1)-(3.3), describe a system of three dependent variables, $C(t), K(t)$, and $I(t)$, which represent tumour volume, cancer carrying capacity volume, and immune volume respectively. Parameter $\alpha$ is the intrinsic tumour growth rate and $K(t)$ is the dynamic cancer carrying capacity with stimulation coefficient $p$ and inhibition coefficient $q$. The dynamic cancer carrying capacity $(K(t))$ is determined by a balance of stimulatory (pro-angiogenic) and inhibitory (anti-angiogenic) terms [17]. The dynamic capacity thus grows 
in response to the growing cancer, which is more biological.

In the dynamic carrying capacity, parameters $a$ and $b$ determine the weight of pro-tumour immune actions contributing to capacity growth. The majority of these signals are assumed to originate from the cancer population, requiring $a$ and $b$ to be small. The weight of immuneproduced tumour-promoting factors (i.e. pro-angiogenic signals) that act to increase the tumours carrying capacity is controlled by $a$, and the weight of immune-produced tumourinhibiting factors (i.e. anti-angiogenic signals) that act to limit the tumours carrying capacity is controlled by $b$. Thus, in this study $a>b$ so that more weight is placed on immune stimulation of cancer carrying capacity than on inhibition. Further it is assumed that $0 \leq a \leq 1$ and $0 \leq b \leq \frac{2}{3}$. For the immune population, $\beta$ is the intrinsic immune growth rate, $K_{I}$ is the constant carrying capacity, and $\rho$ is the cancer-induced immune recruitment.

$$
\begin{aligned}
\frac{d C}{d t} & =\alpha C\left(1-\frac{C}{K}\right) & C(0) & =C_{0} \\
\frac{d K}{d t} & =p(1+I)^{a} C^{1-a}-q K(1+I)^{b} C^{\frac{2}{3}-b} & & K(0)=K_{0} \\
\frac{d I}{d t} & =\beta(I+\rho C)\left(1-\frac{I}{K_{I}}\right) & I(0) & =I_{0}
\end{aligned}
$$

\subsection{Two-Tumour Model}

In the two-tumour scenario, the hypothesis purports that one tumour site will be larger than the other due to competition for pro-tumour inflammatory immune cells. To model this, two sets of the control one-tumour model are coupled together via immune competition.

Let $C_{1}(t), K_{1}(t)$, and $I_{1}(t)$ be the variables describing the big tumour site's tumour volume, cancer carrying capacity volume, and immune volume, respectively. Similarly, $C_{2}(t), K_{2}(t)$, and $I_{2}(t)$, describe the small tumour site's tumour volume, cancer carrying capacity volume, and immune volume, respectively. The model equations are thus: 


$$
\begin{aligned}
\frac{d C_{1}}{d t} & =\alpha C_{1}\left(1-\frac{C_{1}}{K_{1}}\right) & C_{1}(0) & =C_{10} \\
\frac{d K_{1}}{d t} & =p\left(1+I_{1}\right)^{a} C_{1}^{1-a}-q K_{1}\left(1+I_{1}\right)^{b} C_{1}^{\frac{2}{3}-b} & K_{1}(0) & =K_{10} \\
\frac{d I_{1}}{d t} & =\beta\left(I_{1}+\rho C_{1}\right)\left(1-\frac{I_{1}+I_{2}}{K_{I}}\right) & I_{1}(0) & =I_{10} \\
\frac{d C_{2}}{d t} & =\alpha C_{2}\left(1-\frac{C_{2}}{K_{2}}\right) & C_{2}(0) & =C_{20} \\
\frac{d K_{2}}{d t} & =p\left(1+I_{2}\right)^{a} C_{2}^{1-a}-q K_{2}\left(1+I_{2}\right)^{b} C_{2}^{\frac{2}{3}-b} & K_{2}(0) & =K_{20} \\
\frac{d I_{2}}{d t} & =\beta\left(I_{2}+\rho C_{2}\right)\left(1-\frac{I_{1}+I_{2}}{K_{I}}\right) & I_{2}(0) & =I_{20}
\end{aligned}
$$

Nominally, parameter values at each site should be the same as in the control one-tumour model. The coupling of the two sites is introduced by requiring both $I_{1}(t)$ and $I_{2}(t)$ to draw from the same systemic pool of immune carrying capacity, the constant $K_{I}$. This is introduced into equations (3.6) and (3.9). 


\section{Chapter 4}

\section{Model Parameterization}

For the control one-tumour model, some parameters are fixed and the remaining parameters are fitted to our control experimental data. The fixed parameters include $C_{0}, I_{0}, K_{I}$, $a$, and $b$. From the experimental setup, $C_{0}=10^{6}$ cells which is approximately $1 \mathrm{~mm}^{3}$. For the immune population, no initial presence is assumed, $I_{0}=0 \mathrm{~mm}^{3}$, and $K_{I}=1500 \mathrm{~mm}^{3}$, which is a similar size to the maximal control tumour burden exhibited in the data. In this work, $a=0.2$, which allows immune cells to contribute to tumour promotion but requires the majority of stimulation to originate from the tumour itself. Similarly, $b=0.1$ (a value slightly less than $a$ ), limiting the amount of tumour inhibition originating from immune actions.

The remaining model parameters $\left(\alpha, K_{0}, p, q, \beta\right.$, and $\left.\rho\right)$ are determined by fitting the model prediction to the control experimental data. To do this, a simulated annealing algorithm [8] is used with the root mean squared error (RMSE) objective function, defined as

$$
\mathrm{RMSE}=\sqrt{\frac{\sum_{i=1}^{n}\left(C_{i}-C\left(t_{i}\right)\right)^{2}}{n}}
$$

where $C_{i}$ are the control tumour volume measurements on day $t_{i}, C\left(t_{i}\right)$ is the model predicted tumour volume on day $t_{i}$, and $n$ is the total number of data points available.

The simulated annealing algorithm works as follows:

1. Generate a random initial guess for parameter vector $V=\left[\alpha, K_{0}, p, q, \beta, \rho\right]$, numerically compute the solution $C(t)$ by solving the ODE system using the dsolve command in Maple, and calculate the objective function value RMSE

2. One at a time, perturb each parameter to generate a trial vector $V^{\prime}$, numerically compute this new solution $C(t)$ by solving the ODE system using the dsolve command in Maple, and calculate the associated objective function value RMSE'. 
3. Accept this new parameter if the fit is better $\left(R M S E^{\prime}<R M S E\right)$ or accept it sometimes if the fit is worse. Thus acceptance is determined by the probabilistic condition: $p \leq \exp \left(-\frac{1}{T}\left(R M S E^{\prime}-R M S E\right)\right)$, where $p \sim U(0,1)$ is a uniformly distributed random variable.

4. Repeat from 2 until convergence is met

$$
\left(\frac{\left|R M S E_{\mathrm{ave}}-R M S E\right|}{R M S E_{\mathrm{ave}}}<\delta \text { and } \frac{\left|R M S E_{\mathrm{ave}}-R M S E_{\min }\right|}{R M S E_{\mathrm{ave}}}<\delta\right)
$$

with $\delta=0.001$, or until the number of iterations exceeds 35,000 .

The scheme to perturb parameter values and the temperature $T$ are updated as the algorithm moves towards convergence. To estimate the fitted model parameters, the method was run ten times with 35, 000 trials per parameter in each run.

Ten parameter fitting runs were performed (see results in Table 4.1) and the parameter set with the best fit, as measured by RMSE, was chosen (run 4). This best fit is simulated and shown in Figure 4.1. Parameter values were rounded to three significant digits (Final Set) and used in all numerical simulations, unless otherwise stated. Note that all ten runs fit the data well, as seen in Figure 4.2.

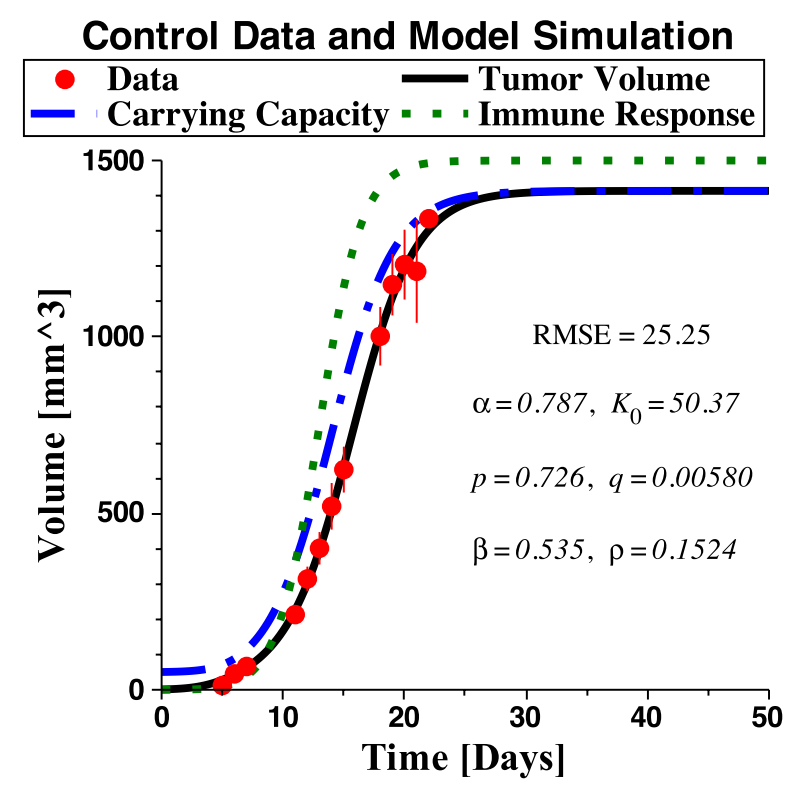

Figure 4.1: The best fit from the simulated annealing algorithm (run 4) numerically plotted against the control experimental data. 
Table 4.1: Simulated annealing results from ten runs of the algorithm to fit the one-tumour model parameters $\alpha, K_{0}, p, q, \beta$, and $\rho$ to control experimental data. The minimum RMSE parameter set (run 4) is highlighted in red. Run 4 parameter values were rounded to three significant digits for use in all numerical simulations (Final Set).

\begin{tabular}{l|c|c|c|c|c|c|c}
\hline & $\alpha$ & $K_{0}$ & $p$ & $q$ & $\beta$ & $\rho$ & RMSE \\
\hline Run 1 & 0.59949 & 121.93 & 1.5545 & 0.012630 & 0.41162 & 0.28871 & 26.502 \\
Run 2 & 0.72289 & 48.101 & 0.93735 & 0.0075513 & 0.41429 & 0.30035 & 25.340 \\
Run 3 & 0.76722 & 40.617 & 0.65666 & 0.0052061 & 0.46719 & 0.74815 & 25.324 \\
Run 4 & 0.78713 & 50.371 & 0.72609 & 0.0057981 & 0.53478 & 0.15241 & 25.259 \\
Run 5 & 0.71542 & 83.812 & 0.71071 & 0.0056548 & 0.65263 & 0.091487 & 25.492 \\
Run 6 & 0.75099 & 42.274 & 0.81046 & 0.0064677 & 0.28287 & 0.99941 & 25.262 \\
Run 7 & 0.72814 & 36.384 & 0.59843 & 0.0046818 & 0.83172 & 0.59145 & 25.689 \\
Run 8 & 0.57498 & 159.27 & 2.4627 & 0.018778 & 0.17165 & 0.62727 & 26.515 \\
Run 9 & 0.63564 & 99.106 & 0.86330 & 0.0069123 & 0.52281 & 0.47301 & 25.440 \\
Run 10 & 0.59944 & 169.16 & 1.2142 & 0.0096719 & 0.43812 & 0.25751 & 25.559 \\
\hline \hline Final Set & 0.787 & 50.4 & 0.726 & 0.00580 & 0.535 & 0.152 & 25.262 \\
\hline
\end{tabular}

Table 4.2: Baseline fitted and fixed parameter values used in all numerical simulations unless otherwise stated.

\begin{tabular}{llll}
\hline & \multicolumn{2}{c}{ Fitted Parameters } & \multicolumn{2}{c}{ Fixed Parameters } \\
\hline$\alpha=0.787$ days $^{-1}$ & $K_{0}=50.4 \mathrm{~mm}^{3}$ & $C_{0}=1 \mathrm{~mm}^{3}$ & $I_{0}=0 \mathrm{~mm}^{3}$ \\
$p=0.726$ days $^{-1}$ & $q=0.00580$ days $^{-1}\left(\mathrm{~mm}^{3}\right)^{-\frac{2}{3}}$ & $a=0.2$ & $b=0.1$ \\
$\beta=0.535$ days $^{-1}$ & $\rho=0.152$ & $K_{I}=1500 \mathrm{~mm}^{3}$ & \\
\hline
\end{tabular}

In summary, the values of all model parameters, both fixed and fitted, used as a baseline in all future simulations and analyses of this thesis are listed in Table 4.2. 


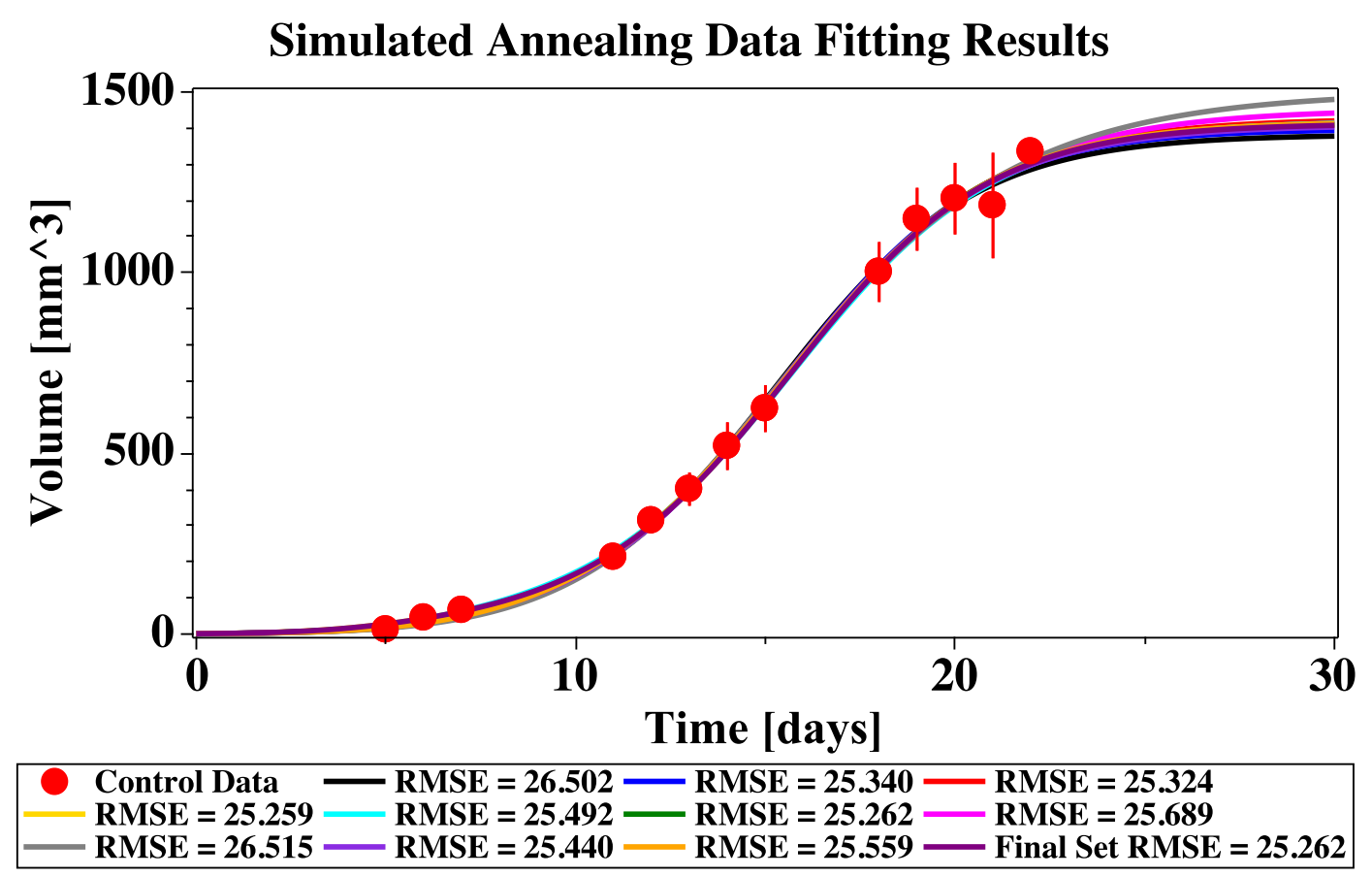

Figure 4.2: Ten runs of the simulated annealing algorithm produced ten parameter sets that all fit the control data equally well (see Table 4.1). The set with the smallest RMSE, rounded to three significant digits, was chosen to work with in all future simulations (Final Set in Table 4.1). 


\section{Chapter 5}

\section{Model Analysis and Parameter Sensitivity}

\subsection{Model Steady State Analyses}

To explore the steady-state of the control one-tumour model, the equilibrium conditions are found for equations (3.1)-(3.3). Three equilibrium points exist in the dynamical system, two cancer-free points $(C, K, I)=(0,0,0),(C, K, I)=\left(0,0, K_{I}\right)$, and one cancerous point $(C, K, I)=\left(K_{c}^{e q}, K_{c}^{e q}, K_{I}\right)$, where $K_{c}^{e q}=\left[\frac{p}{q}\left(1+K_{I}\right)^{a-b}\right]^{\frac{2}{3}+a-b}$.

The two-tumour model, equations (3.4)-(3.9), has five possible equilibrium points as follows: two cancer-free points

$$
\begin{aligned}
& 1\left(C_{1}, K_{1}, I_{1} ; C_{2}, K_{2}, I_{2}\right)=(0,0,0 ; 0,0,0) \\
& 2\left(C_{1}, K_{1}, I_{1} ; C_{2}, K_{2}, I_{2}\right)=\left(0,0, I_{1}^{e q} ; 0,0, I_{2}^{e q}\right),
\end{aligned}
$$

and three cancerous points

$3\left(C_{1}, K_{1}, I_{1} ; C_{2}, K_{2}, I_{2}\right)=\left(K_{C 1}^{e q}, K_{C 1}^{e q}, I_{1}^{e q} ; 0,0,0\right)$,

$4\left(C_{1}, K_{1}, I_{1} ; C_{2}, K_{2}, I_{2}\right)=\left(0,0,0 ; K_{C 2}^{e q}, K_{C 2}^{e q}, I_{2}^{e q}\right)$, and

$5\left(C_{1}, K_{1}, I_{1} ; C_{2}, K_{2}, I_{2}\right)=\left(K_{C 1}^{e q}, K_{C 1}^{e q}, I_{1}^{e q} ; K_{C 2}^{e q}, K_{C 2}^{e q}, I_{2}^{e q}\right)$

where $I_{1}^{e q}+I_{2}^{e q}=K_{I}, K_{C 1}^{e q}=\left[\frac{p}{q}\left(1+I_{1}^{e q}\right)^{a-b}\right]^{\frac{1}{\frac{2}{3}+a-b}}$, and $K_{C 2}^{e q}=\left[\frac{p}{q}\left(1+K_{I}-I_{1}^{e q}\right)^{a-b}\right]^{\frac{1}{3}+a-b}$.

Parameters $a, b, p$, and $q$ figure prominently in the equilibrium sizes of the tumour volumes. Since parameters $a$ and $b$ are fixed in this work, the effect of these parameters on tumour equilibrium size is studied. Figure 5.1a shows the effect of increasing $a$ on $K_{C}^{e q}$, the control 


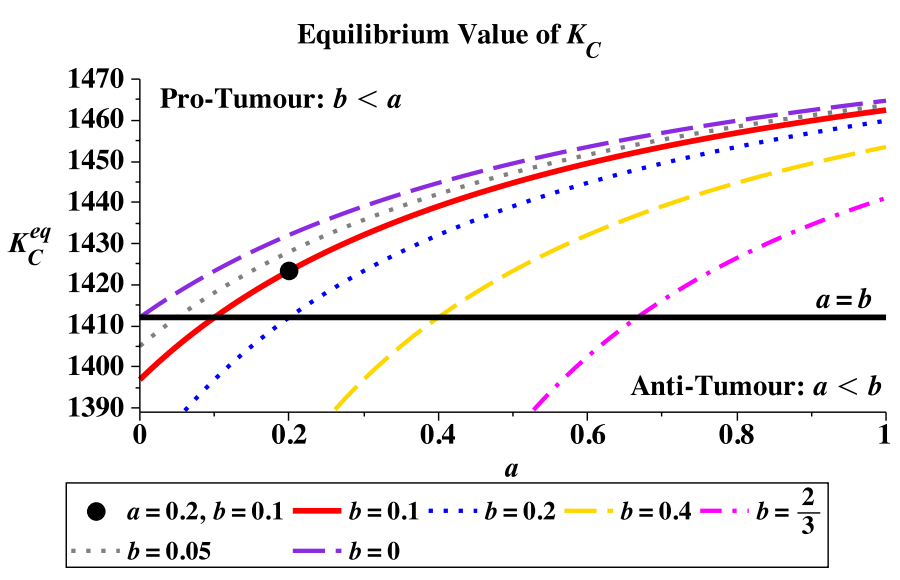

(a) Dependence of $K_{C}^{e q}$ on $a$

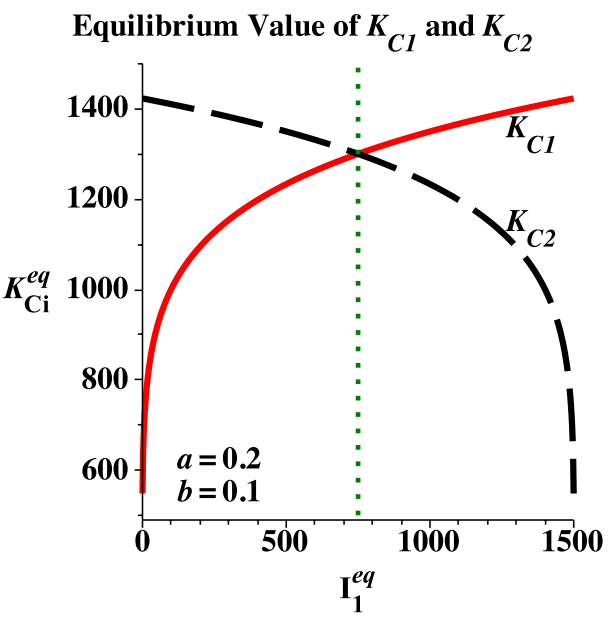

(b) Equilibrium value trade-offs

Figure 5.1: The dependence of equilibrium tumour volume, $K_{C}^{e q}$, in the control model, on parameters $a$ and $b$, shown in (a); and the dependence of equilibrium tumour volumes $K_{C 1}^{e q}$, and $K_{C 2}^{e q}$, of the two-tumour model, on immune equilibrium size $I_{1}^{e q}$, shown in (b).

tumour equilibrium size, for various values of parameter $b$. Overall, when $a$ is increased from $a=b$ to $a=1$, the final tumour size increases less than $4 \%$, suggesting that the values of $a$ and $b$ chosen in this work do not significantly affect the results. That is, $K_{c}^{e q}$ is robust to changes in $a$ and $b$.

The competition for immune recruitment between the two sites in the two-tumour model determines equilibrium tumour size for each site. Figure 5.1b shows that unless there is an extreme difference in equilibrium immune sizes (i.e. $I_{1}^{e q} \gg I_{2}^{e q}$ or $I_{2}^{e q} \ll I_{1}^{e q}$ ), the two sites will have comparable volumes (less than a $30 \%$ difference). Recall, however, that this equilibrium analysis does not demonstrate behaviours for short time. This analysis suggests that even though one site may have a faster growth rate than the other site at early times, for late times the two tumour volumes will be comparable.

\subsection{Parameter Sensitivity}

A parameter sensitivity analysis is used to determine the local dependency of model predictions on parameter values. In the control one-tumour model, there are both fixed and fitted parameters. In the analysis, each parameter value is varied by $20 \%$ up or down from the value listed in Table 4.2, and the relative change in tumour volume at day 15 is reported in Figure 5.2. Note that for the fitted parameters, tumour growth rate $\alpha$ and cancer carrying capacity stimulation 
coefficient $p$ are the most influential to tumour volume. For fixed parameters, initial tumour volume $C_{0}$ is the most influential, but this value is determined by the experimental setup.

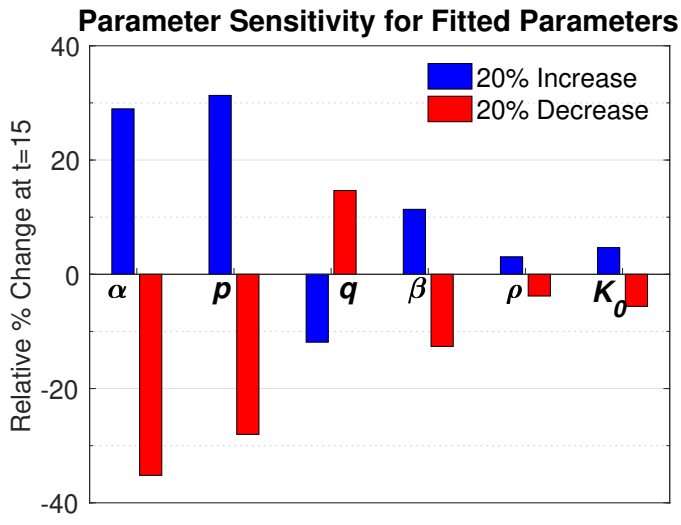

(a) Fitted Parameters

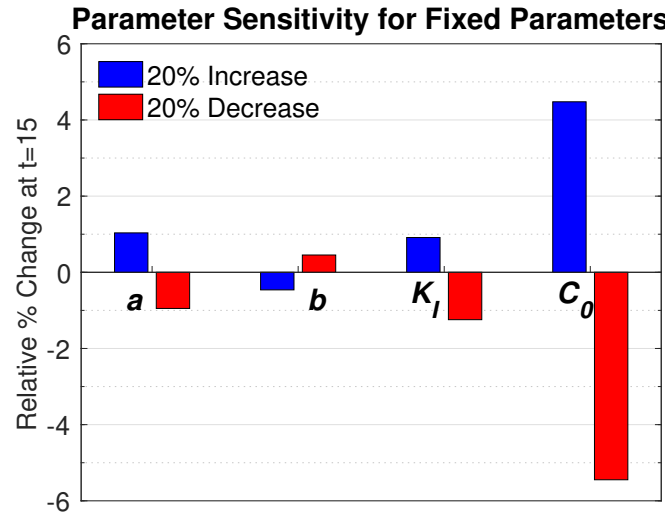

(b) Fixed Parameters

Figure 5.2: Each parameter value is varied by $20 \%$ up and down and the relative change in tumour volume at day 15 is reported.

Fitted model parameters $q, \beta, \rho$, and $K_{0}$ have less influence on tumour size on day 15 compared to $\alpha$ and $p$. Similarly, the fixed model parameters $a, b$, and $K_{I}$ have less influence compared to $C_{0}$. This result is important as the values for $a, b$, and $K_{I}$ were chosen based on arguments presented in Chapter 3.2 to simplify the parameterization process. 


\section{Chapter 6}

\section{Numerical Simulations and Results}

This chapter presents several numerical simulations of the two-tumour model designed to test potential causative mechanisms related to the proposed biological hypothesis. All model simulations are computed using the ODE45 command in MATLAB.

\subsection{Single Variable Mechanisms}

Using the two-tumour model, equations (3.4)-(3.9), single causative mechanisms are tested to determine if differences in the corresponding test parameter value can explain the observed growth rate differences. To do this, all parameter values are set to the nominal values listed in Table 4.2 except the one parameter that corresponds to the test mechanism. This test parameter is then allowed to vary, and the value generating the best fit to the two tumour data (as measured by RMSE) is found.

First the initial tumour size at the smaller side (here assumed to be $C_{2}(0)=C_{20}$ ) is tested. Biologically, this corresponds to the second injection containing less viable (less healthy and less proliferative) cells compared to the first injection. Mathematically, this is described by $C_{20} \leq C_{10}$, and this condition is fit to the experimental data by defining $C_{20}=\gamma C_{10}$, where $\gamma \in[0,1]$ and $C_{10}=1 \mathrm{~mm}^{3}$. The best fit, as shown in Figure $6.1 \mathrm{a}$ is obtained when $\gamma=0.053$. This corresponds to the second injection $\left(C_{20}\right)$ containing only $5 \%$ of the viable cells in the first injection $\left(C_{10}\right)$. This extreme difference required by the model to explain the data is not acceptable as a potential mechanism because it is not biologically feasible given the experimental setup. Thus, this single mechanism is rejected as a potential causative mechanism explaining the different growth rates.

Next, immune growth rate is tested as a potential causative mechanism. Biologically, this corresponds to different intrinsic growth rates between the two sides, due to different combina- 
tions and levels of signaling factors (cytokines) present at the two sites. Mathematically, this is described as $\beta_{2} \leq \beta_{1}$, and this condition is fit to the experimental data by defining $\beta_{2}=\gamma \beta_{1}$, where $\gamma \in[0,1]$ and $\beta_{1}=0.535$ from Table 4.2. The best fit, as shown in Figure $6.1 \mathrm{~b}$ is obtained when $\gamma=0.228$. This corresponds to the small tumour site having an intrinsic growth rate of $\beta_{2}=0.122$, potentially due to a decrease in immune recruitment and proliferative signals at that site. This difference required by the model to explain the data is not acceptable as a potential mechanism because the difference is large and thus determined to be not biologically feasible. Thus, this single mechanism is rejected as a potential causative mechanism explaining the different tumour growth rates.

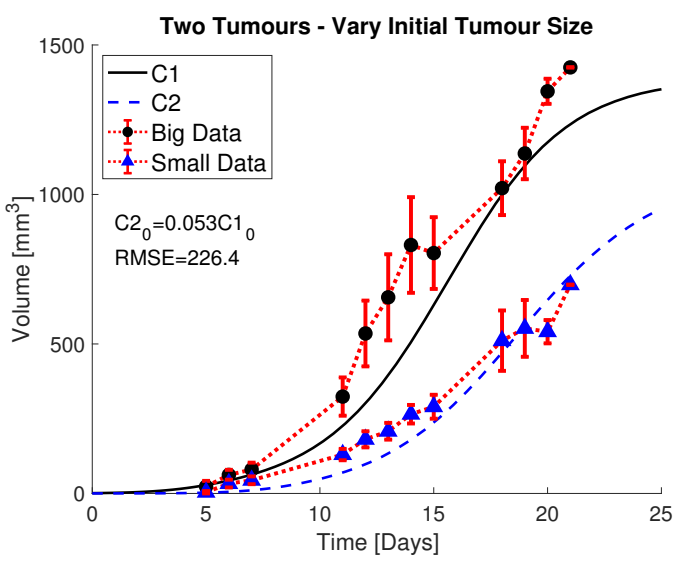

(a) Vary initial tumour size

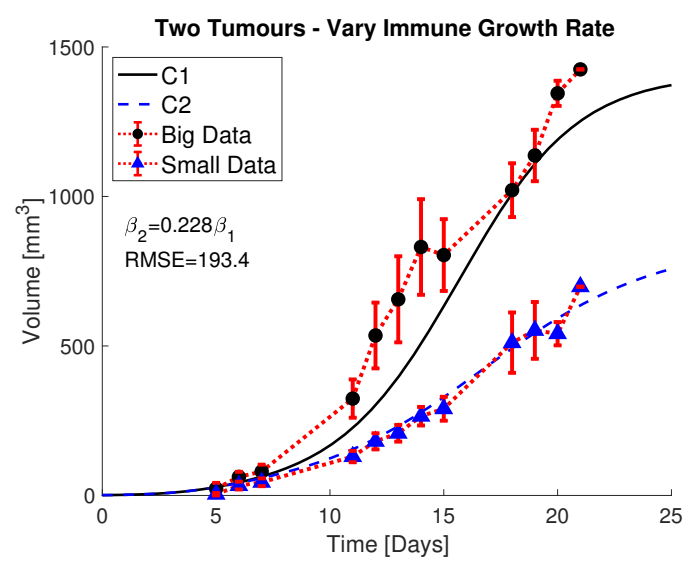

(b) Vary immune growth rate

Figure 6.1: Two-tumour simulation testing differences in initial tumour size (a) and intrinsic immune growth rate $(\mathrm{b})$.

Next, initial immune size is tested as a potential causative mechanism. Nominally, in all simulations, no initial immune presence is assumed. Here, however, initial immune presence is tested to determine if increasing the initial condition can explain the different tumour growth rates. Biologically, this corresponds to one site already containing inflammatory immune cells potentially due to injury (bruise or bite for example) prior to injection. To fit the model prediction to the data, $I_{20}=0$ and $I_{10}$ is allowed to increase positively. The best fit, as shown in Figure $6.2 \mathrm{a}$ is obtained when $I_{10}=103 \mathrm{~mm}^{3}$, which is a large initial presence compared to the other site. This extreme difference required by the model to fit the data is determined to be not acceptable, and thus, this single mechanism is rejected.

Finally, immune recruitment is tested as a potential mechanism. Biologically, this corresponds to the strength with which the local cancer presence is attracting immune cells, and is determined by the signalling factors they produce. Mathematically, this is describe by $\rho_{2} \leq \rho_{1}$, 


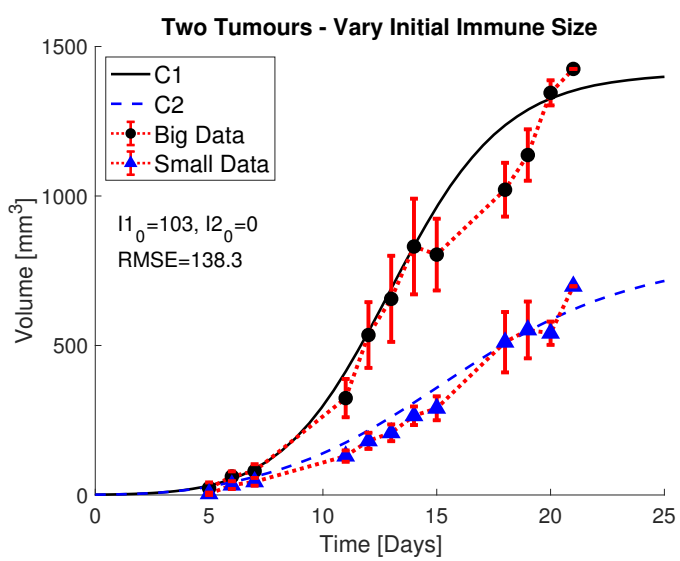

(a) Vary initial immune size

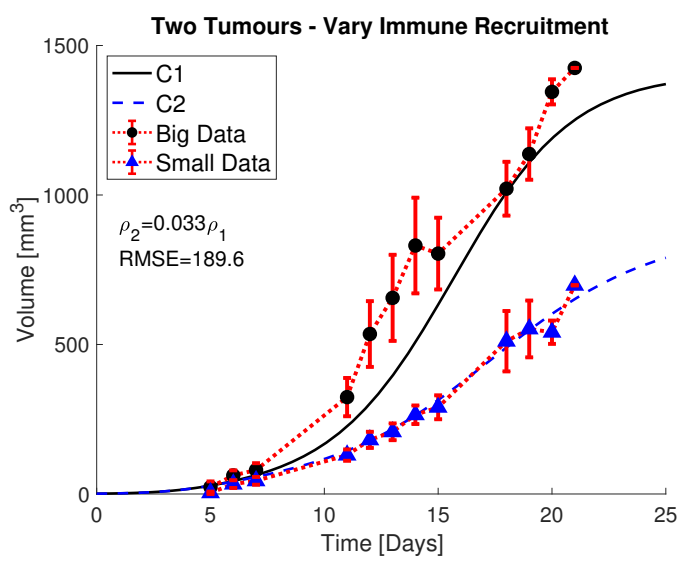

(b) Vary immune recruitment

Figure 6.2: Two-tumour simulation testing differences in initial immune size (a) and immune recruitment (b).

and this condition is fit to the experimental data by defining $\rho_{2}=\gamma \rho_{1}$, where $\gamma \in[0,1]$ and $\rho_{1}=0.152$ from Table 4.2. The best fit, as shown in Figure $6.2 \mathrm{~b}$ is obtained when $\gamma=0.033$. This corresponds to the smaller tumour site having an immune recruitment parameter of $\rho_{2}=0.005$, which is determined to be unacceptably small. Thus, this single mechanism is rejected as a potential causative mechanism explaining the different tumour growth rates.

All four of the single parameter mechanisms tested above have been rejected because they require extreme differences between the two sites which are determined to be unacceptable and not biologically feasible. Thus, combinations of these parameter values will now be tested.

\subsection{Combination Variable Mechanisms}

The first combination of mechanisms tested, includes the four single parameters described in the previous section $\left(C_{20}, \beta_{2}, I_{10}\right.$, and $\left.\rho_{2}\right)$. The result of allowing all four of these parameters to vary at once is shown in Figure $6.3 \mathrm{a}$. The best fit requires $C_{20}=0.3 \mathrm{~mm}^{3}, \beta_{2}=0.9 \beta_{1}, I_{10}=15$ $\mathrm{mm}^{3}$, and $\rho_{2}=0.9 \rho_{1}$. Although the immune growth rate $\left(\beta_{2}\right)$, the immune recruitment $\left(\rho_{2}\right)$, and even the initial immune presence $\left(I_{10}\right)$ are all acceptably small deviations off their baseline values, the initial cancer presence $\left(C_{20}\right)$ is not. The best fit requires the second tumour injection to contain only $30 \%$ of the number of viable cells in the first injection. This is determined to be not biologically feasible and this combination is thus rejected.

Next, the initial carrying capacity of each site is tested as a potential causative mechanism. To fit the model prediction to the data, both $K_{10}$ and $K_{20}$ are allowed to increase positively 
from zero. Recall that the baseline value for $K_{0}=50.4 \mathrm{~mm}^{3}$ (see Table 4.2). To fit the data, it is expected that $K_{20} \leq K_{10}$, and in fact the best fit requires $K_{10}=204.3 \mathrm{~mm}^{3}$ and $K_{20}=2.1$ $\mathrm{mm}^{3}$, as shown in Figure 6.3b. This corresponds to the first site initially having a much larger capability to support tumour growth than the second site, potentially due to an increased vasculature and/or inflammatory presence. The difference in initial capacity values required by the model to fit the data, however, is determined to be too large, and thus this test case is rejected.

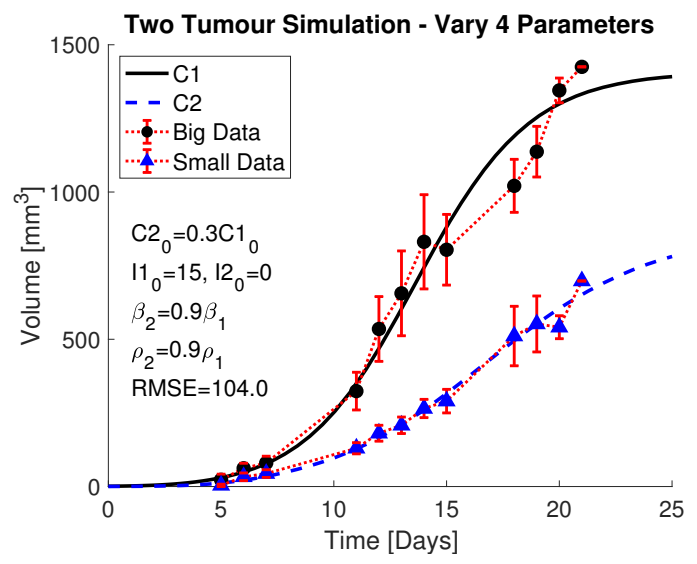

(a) Vary four parameters

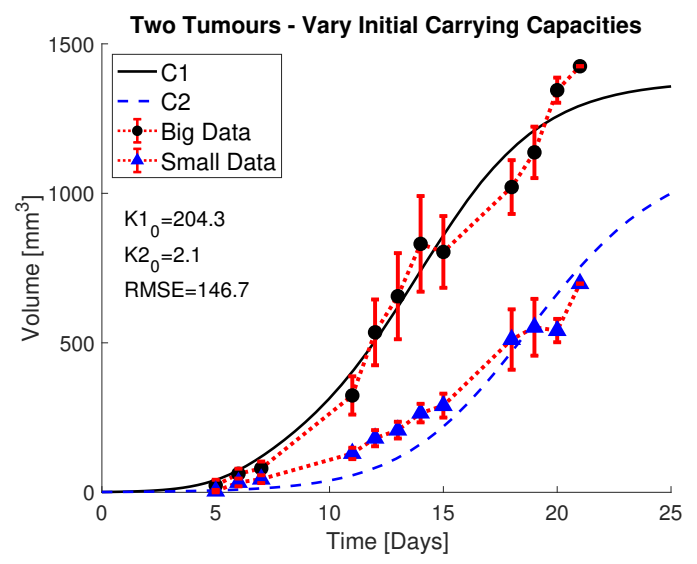

(b) Vary initial carrying capacity

Figure 6.3: Two-tumour simulation varying either the four parameters $C_{20}, I_{10}, \beta_{2}$, and $\rho_{2}$ in (a), or varying the initial carrying capacities $K_{10}$ and $K_{20}$ in (b).

The next combination of mechanisms tested involves all six parameters described so far, $\left(C_{20}, I_{10}, \beta_{2}, \rho_{2}, K_{10}\right.$, and $\left.K_{20}\right)$. The result of allowing all six of these parameters to vary at once is shown in Figure 6.4a. The best fit requires $C_{20}=0.5 \mathrm{~mm}^{3}, I_{10}=1 \mathrm{~mm}^{3}, \beta_{2}=\beta_{1}$, $\rho_{2}=0.1 \rho_{1}, K_{10}=146 \mathrm{~mm}^{3}$, and $K_{20}=53 \mathrm{~mm}^{3}$. This best fit has an RMSE $=99.5$, which is the smallest achieved so far. Note that the immune growth rate $\beta_{2}=\beta_{1}$ and the initial immune presence $I_{10} \approx I_{20}$. Further, immune recruitment $\rho_{2}$ is only $10 \%$ of $\rho_{1}$, however, the sensitivity analysis shows that the model output is not very sensitive to $\rho$, and so this difference may be acceptable. Initial cancer presence at site two, $C_{20}$, is only $50 \%$ of that associated with site one, which is again deemed to be unacceptable and not biologically feasible. This fit also requires a large difference between the initial carrying capacities of the two sites, which is also not acceptable. These large differences in initial cancer presence and carrying capacities require the rejection of this combination of mechanisms.

The last combination of mechanisms tested, are those associated with a pre-inflamed site prior to injection. Parameters that describe this environment are $I_{10}, I_{20}, K_{10}$, and $K_{20}$. Note 


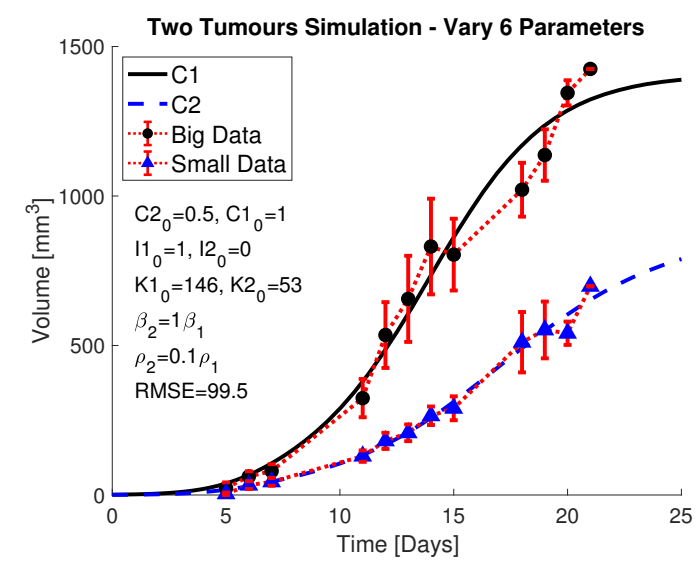

(a) Vary six parameters

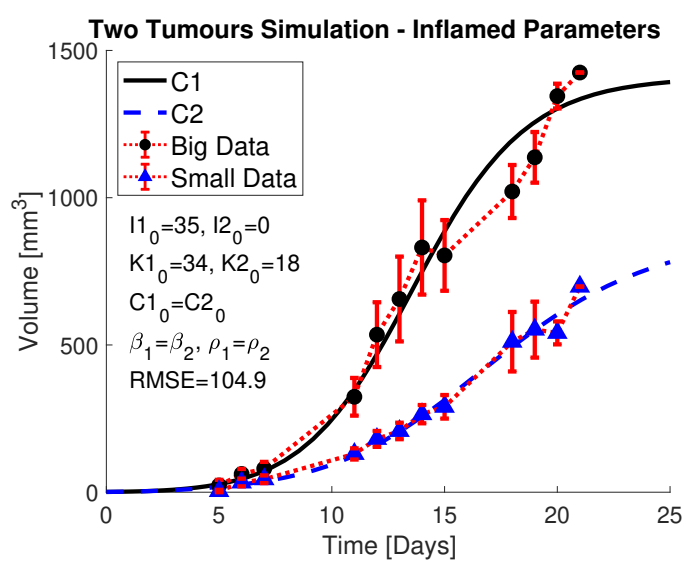

(b) Vary inflamed parameters

Figure 6.4: Two-tumour simulation varying either the six parameters $C_{20}, I_{10}, \beta_{2}, \rho_{2}, K_{10}$, and $K_{20}$ in (a), or varying the pre-inflamed parameters $I_{10}, I_{20}, K_{10}$, and $K_{20}$ in (b).

that other parameters are fixed to be equal between the two sites: $C_{10}=C_{20}, \beta_{1}=\beta_{2}$, and $\rho_{1}=\rho_{2}$ at the baseline values listed in Table 4.2. The result of allowing these pre-inflamed parameters to vary is shown in Figure $6.4 \mathrm{~b}$. The best fit requires $I_{10}=35 \mathrm{~mm}^{3}, I_{20}=0 \mathrm{~mm}^{3}$, $K_{10}=34 \mathrm{~mm}^{3}$, and $K_{20}=18 \mathrm{~mm}^{3}$. The differences between the two sites in parameter values are not large and thus deemed acceptable. Further, the fit is visually good, and the RMSE $=104.9$, which is not the best achieved in this work, but is still a very good fit. The biological mechanisms underlying these four parameters correspond to one site being pre-inflamed prior to cancer cell injection. This could be caused by a bruise or injury to the site before the experiment started which is a possibility. This pre-inflamed combination of mechanisms is thus accepted as the best prediction of the model to fit the experimental data within the framework of the proposed biological hypothesis. 


\section{Chapter 7}

\section{Discussion and Conclusions}

Experimental data shows that when two tumours are injected simultaneously, their growth rates may differentiate causing one slow and one fast growing tumour. This thesis presents unbalanced accumulation of tumour-promoting inflammation as a potential biological explanation for this growth rate separation. It then develops and analyzes a mathematical model designed to test this hypothesis. The model formulation involves a multi-compartment ODE system capturing tumour volume, cancer carrying capacity, and immune volume at each of the two injection sites.

To explore the shape of the experimental data, exponential and logistic growth models were fit to the data, assuming either a fixed or variable initial condition $\left(C_{0}=10^{6}\right.$ cells $\left.\approx 1 \mathrm{~mm}^{3}\right)$. The best fit to the data was the 3-parameter model, the logistic model with an initial condition fit to the data. Of the two 2-parameter models considered, the logistic model also provided a better fit compared to the exponential model. The logistic growth model was thus used to describe tumour growth in the developed ODE system.

The model is formulated such that the cancer volume is assumed to grow according to a generalized logistic law that is mechanistically accentuated by tumour promoting inflammation altering the dynamic carrying capacity. The hypothesis explored here suggests that one tumour site may be larger than the other because of unbalanced accumulation of tumour-promoting immune cells. Competition for immune recruitment between the two sites determines the local immune presence at each site, but their combined size is limited by the fixed immune capacity $K_{I}$.

Some model parameter values were fixed to simplify the parameterization process. Specifically, $a=0.2$ and $b=0.1$ were chosen such that more weight of immune action was placed on tumour promotion rather than inhibition $(a>b)$, and the majority of cancer growth was stimulated by the tumour itself ( $a$ and $b$ small). Additionally, $K_{I}=1500 \mathrm{~mm}^{3}$ was chosen such that the immune response obtained a similar maximal size as the control tumour burden. 
Nominally $C_{0}=1 \mathrm{~mm}^{3}$ as this was the experimental setup, and and $I_{0}=0 \mathrm{~mm}^{3}$ as no initial immune presence was assumed. Model parameterization was then performed using the control (one-tumour) model and data. The remaining model parameters $\left(\alpha, p, q, \beta, \rho\right.$, and $\left.K_{0}\right)$ were estimated using a simulated annealing algorithm. Of ten runs, the parameters obtaining the smallest RMSE were chosen to work with in all simulations.

A numerical parameter sensitivity analysis was performed by varying each parameter value by 20 percent (up and down) and reporting the relative change in tumour volume on day 15. Of the fitted parameters, intrinsic tumour growth rate $(\alpha)$ and cancer carrying capacity stimulation coefficient $(p)$ were the most influential to tumour volume. For fixed parameters, initial tumour volume $C_{0}$ was most influential but this value is determined by the experimental setup. Importantly, fixed parameters $a$ and $b$ were found to be some of the least significant parameters to tumour volume, which supports the choice to fix these values prior to data fitting.

Numerical simulations of the two-tumour model were performed to test the biological hypothesis. To do this, either single parameters or combinations of parameters were fit to the two-tumour data. RMSE was used to measure the goodness-of-fit for each simulation. The best fit was found by perturbing all six considered parameters / mechanisms. However, due to biological constraints, it is suggested here that the most biologically probable combination of parameters that enable the model to fit the data are the pre-inflamed parameters: $I_{10}, I_{20}$, $K_{10}$, and $K_{20}$. This combination of parameters fit to the two-tumour data describes a situation where one site has a slightly larger initial immune presence and a slightly elevated initial cancer carrying capacity than the other site. These slight differences between the two sites allow the model to predict one fast and one slow growing tumour in a biologically feasible scenario.

Biologically, this work highlights the significant influence that inflammation can have on tumour growth. The model demonstrates that inflammatory cells have the potential to alter growth dynamics significantly, and in a systemic (whole-host) manner. This extends not just to multiple tumour burdens, such as in metastatic conditions, but also to the different growth rates observed within the same tumour type across individuals (inter-patient variability). Inflammation is a hallmark of cancer, associated with all cancer types, and it provides a simple explanation for the experimental data focused on in this study, compared to concomitant resistance or other mechanisms.

\subsection{Future Work}

In this thesis, I presented a mathematical model to describe the effects of localized inflammatory immune recruitment and competition between two cancer sites on tumour growth rates, using a multi-compartment ODE system. Further work could incorporate concomitant resistance 
mechanisms of angiogenesis inhibition or proliferation inhibition into the model formulation developed here. Then, the concomitant resistance mechanisms could be compared to the inflammation mechanism within the same model framework, to determine which mechanisms best describe the two-tumour experimental data. Furthermore, the development of a PDE model from this ODE framework would expand on the spatial aspects of the immune competition and allow further numerical testing of the proposed hypothesis. 


\section{References}

[1] J. Adam and N. Bellomo. A survey of models for tumor immune system dynamics. Birkhuser, 1997.

[2] M. Al-Tameemi, M. Chaplain, and A. d'Onofrio. Evasion of tumours from the control of the immune system: consequences of brief encounters. Biol Direct, 7(1):31, 2012.

[3] E. Angelis and B. Lods. On the kinetic theory for active particles: A model for tumor immune system competition. Math Comput Model, 47:196-209, 2008.

[4] F. Balkwill and A. Mantovani. Inflammation and cancer: back to Virchow? Lancet, $357: 539-45,2002$.

[5] E. Bashford, J. Murray, and M. Haaland. General results of propagation of malignant new growths. Cancer Res, 3:262-8, 1908.

[6] N. Bellomo and M. Delitala. From the mathematical kinetic, and stochastic game theory to modelling mutations, onset, progression and immune competition of cancer cells. Physics of Life Reviews, 5(4):183-206, 2008.

[7] S. Benzekry, C. Lamont, D. Barbolosi, L. Hlatky, and P. Hahnfeldt. Mathematical modeling of tumor-tumor distant interactions supports a systemic control of tumor growth. Cancer Res, 77(18):5183-5193, 2017.

[8] A. Corana, M. Marchesi, C. Martini, and S. Ridella. Minimizing multimodal functions of continuous variables with the "simulated annealing" algorithm. ACM Trans Mathematical Software, 13(3):262-280, September 1987.

[9] L. de Pillis, A. Radunskaya, and C. Wiseman. A validated mathematical model of cell mediated immune response to tumor growth. Cancer Res, 65(17):7950-7958, 2005.

[10] C. DeLisi and A. Rescigno. Immune surveillance and neoplasia I. A minimal mathematical model. Bull Math Biol, 39:201-221, 1977. 
[11] A. d'Onofrio. A general framework for modeling tumor-immune system competition and immunotherapy: mathematical analysis and biomedical inferences. Phys D, 208(3-4):220$235,2005$.

[12] A. d'Onofrio and A. Ciancio. Simple biophysical model of tumor evasion from immune system control. Phys Rev E, 84(3), 2011.

[13] G. Dunn, L. Old, and R. Schreiber. The three E's of cancer immunoediting. Annu Rev Immunol, 22:329-60, 2004.

[14] R. Eftimie, J. Bramson, and D. Earn. Interactions between the immune system and cancer: a brief review of non-spatial mathematical models. Bull Math Biol, 73(1):2-32, 2010.

[15] H. Enderling, L. Hlatky, and P. Hahnfeldt. Immunoediting evidence of the multifaceted role of the immune system in self metastatic tumor growth. Theor Biol Med Model, 9:31, 2012 .

[16] S. I. Grivennikov, F. R. Greten, and M. Karin. Immunity, inflammation, and cancer. Cell, 140(6):883-899, March 2010.

[17] P. Hahnfeldt, D. Panigraphy, J. Folkman, and L. Hlatky. Tumor development under angiogenic signaling: a dynamical theory of tumor growth, treatment response, and postvascular dormancy. Cancer Res, 59(19):4770-5, 1999.

[18] D. Kirschner and J. Panetta. Modeling immunotherapy of the tumor immune interaction. J Math Biol, 37(3):235-252, 1998.

[19] V. A. Kuznetsov, I. A. Makalkin, M. A. Taylor, and A. S. Perelson. Nonlinear dynamics of immunogenic tumors: parameter estimation and global bifurcation analysis. Bull Math Biol, 56(2):295-321, 1994.

[20] R. Lefever and W. Horsthemke. Bistability in fluctuating environments. implications in tumor immunology. Bull Math Biol, 41:469-490, 1979.

[21] W. Lin and M. Karin. A cytokine-mediated link between innate immunity, inflammation, and cancer. J Clin Invest, 117(5):1175-83, 2007.

[22] A. Matzavinos, M. Chaplain, and V. Kuznetsov. Mathematical modelling of the spatio temporal response of cytotoxic tlymphocytes to a solid tumour. Math Med Biol, 21(1):1$34,2004$. 
[23] S. Rakoff-Nahoum. Why cancer and inflammation? Yale J Biol Med, 79(3-4):123-130, 2006 .

[24] T. Roose, S. Chapman, and P. Maini. Mathematical models of avascular tumor growth. SIAM Rev, 49(2):179-208, 2007.

[25] R. Ruggiero, J. Bruzzo, P. Chiarella, O. D. Bustuoabad, R. P. Meiss, and C. D. Pasqualini. Concomitant tumor resistance: The role of tyrosine isomers in the mechanisms of metastases control. Cancer Res, 72, 2012.

[26] H. Shen, X. Yu, F. Yang, Z. Zhang, J. Shen, J. Sun, S. Choksi, S. Jitkaew, and Y. Shu. Reprogramming of normal fibroblasts into cancer-associated fibroblasts by miRNAs-mediated CCL2/VEGFA signaling. PLOS Genetics, 2016.

[27] K. Wilkie. A review of mathematical models of cancer immune interactions in the context of tumor dormancy. Adv Exp Med Biol, 734:201-234, 2013.

[28] K. Wilkie and P. Hahnfeldt. Tumor immune dynamics regulated in the microenvironment inform the transient nature of immune induced tumor dormancy. Cancer Res, 73(12):3534$3544,2013$.

[29] K. P. Wilkie and P. Hahnfeldt. Modeling the dichotomy of the immune response to cancer: Cytotoxic effects and tumor-promoting inflammation. Bull Math Biol, 79(6):1426-48, 2017. 